\title{
A Nonlinear Programming Approach For a Fuzzy queue with an unreliable server
}

\author{
Dr.V. Ashok Kumar \\ Shinas College of Technology, Oman \\ E-mail: vaashok1963@rediffmail.com
}

Keywords : Fuzzy sets, Nonlinear Programming, Queue, Solution algorithm, Unreliable server.

\begin{abstract}
The aim of this paper is to develop the membership functions of the system characteristic of a queuing g model with an unreliable server, in which the arrival rate, service rate, breakdown rate and repair rate are all fuzzy numbers. The $\alpha$-cut approach is used to transform a fuzzy queue with an unreliable server into a family of conventional crisp queues with an unreliable server. By using membership functions, a set of parametric nonlinear programmes are developed to describe the family of crisp queues with an unreliable server. An efficient algorithm is developed to find the optimal solutions at or different possibility level $\alpha$. Numerical examples are solved successfully. Since the system characteristics being expressed and governed by membership functions, more information is provided for the management.
\end{abstract}

\section{INTRODUCTION}

Most studies on queuing systems use "Perfect" (reliable) servers. However in real life systems, the server may experience an unpredictable breakdowns. Therefore, queuing models with server breakdowns provide a realistic representation of such systems. Regarding queuing model with server breakdown, Gaver [3] first proposed an ordinary $\mathrm{M} / \mathrm{G} / \mathrm{I}$ queuing system with interrupted service and priorities. His system was extended to the GI/G/I case by Sengupta [8]. Li et al. [6] and Wang [10] investigated the behaviour of the unreliable server, and the effect the server breakdowns and repairs in the $\mathrm{M} / \mathrm{G} / \mathrm{I}$ queuing models and he investigated the controllable $\mathrm{M} / \mathrm{H}_{\mathrm{K}} / \mathrm{I}$ queuing systems with an unreliable server recently.

In the above literature, the inter arrival times, service times of customers, breakdown and repair times follow certain probability distributions. However in real-word applications, the statistical applications may be obtained subjectively such as "very fast", "fast", "slow" \& "approximately". In otherwords, these system parameters are more possibilistic as well as probabilistic. Thus fuzzy queues are potentially useful and are much more realistic than the commonly used crisp queues. The fuzzy queues with an unreliable server have wider applications than the commonly used crisp queues with an unreliable server.

It should be noted that, all the previous research on the fuzzy queuing models is focused on one (or) two fuzzy variables. In this paper, we develop an approach that can provide the system characteristics of fuzzy queues with an unreliable server with four fuzzy variables, namely fuzzified exponential arrival rate, service rate, breakdown rate and repair rate. Through the $\square$-cuts and Zadch's extension principle, we transform the fuzzy queue with an unreliable server into a family of crisp queues with an unreliable server. As $\alpha$-varies, the family of crisp queues are then described and solved by parametric nonlinear programming.

For notational convenience our model in this paper will be denoted by FM/FM(FM,FM)/I where the first to the fourth FM represents the fuzzified exponential arrival, service, breakdown and repair rate respectively. In contrast $\mathrm{M} / \mathrm{M}(\mathrm{M}, \mathrm{M}) / \mathrm{I}$ model represents the exponential inter-arrival (Poisson), service, breakdown and repair rates respectively. 


\section{FUZZY QUEUES WITH AN UNRELIABLE SERVER}

\subsection{M/M (M, M) / I queues}

Consider a queuing system with an unreliable server and two different types of breakdowns. In type I, the server may breakdown when there is atleast one customer in the system and in type II, the server may breakdown even if there are no customers in the system. It is assumed that the customers arrive at a single server facility as a Poisson process with rate $\lambda$, the service times as an exponential distribution with rate $\mu$, the server may have a breakdown following poisson process with rate $\sigma$, and the repair follows an exponential distribution with rate $\beta$. Let $\mathrm{E}$ and $\mathrm{F}$ represents the probability that the system is idle in type I and type II respectively.

$$
\begin{aligned}
& \mathrm{E}=\frac{\beta \mu-\lambda(\sigma+\beta)}{\beta \mu} \\
& \mathrm{F}=\frac{\beta \mu-\alpha(\sigma+\beta)}{\mu(\sigma+\beta)}
\end{aligned}
$$

In steady-state, it is required that $0<\frac{\beta \mu-\lambda(\sigma+\beta)}{\beta \mu}<1$ and $0<\frac{\beta \mu-\alpha(\sigma+\beta)}{\mu(\sigma+\beta)}<1$.

$$
1-\frac{\lambda}{-}
$$

Also it is noted that by letting $\sigma=0$ in (1) yields the result $\mu$, which coincides with that of the $\mathrm{M} / \mathrm{M} / \mathrm{I}$ queuing system with a reliable server (see [4]).

\subsection{FM / FM (FM, FM) / I queues}

Suppose the arrival rate $\lambda$, service rate $\mu$, breakdown rate $\sigma$, repair rate $\beta$ are approximately known and can be represented by fuzzy sets $\tilde{\lambda}, \tilde{\mu}, \widetilde{\sigma}$ and $\widetilde{\beta}$ respectively. Let $\eta_{\tilde{\lambda}}(x), \eta_{\tilde{\mu}}(y), \eta_{\widetilde{\sigma}}(\mathrm{u})$ and $\eta_{\tilde{\beta}}(v)$ denote the membership functions of $\tilde{\lambda}, \tilde{\mu}, \tilde{\sigma}$ and $\tilde{\beta}$ respectively.

We've the following

$$
\begin{aligned}
& \tilde{\lambda}=\left\{\left(\mathrm{x}, \eta_{\tilde{\lambda}}(\mathrm{x})\right) / \mathrm{x} \in \mathrm{X}\right\} \\
& \tilde{\mu}=\left\{\left(\mathrm{y}, \eta_{\tilde{\mu}}(\mathrm{y})\right) / \mathrm{y} \in \mathrm{Y}\right\} \\
& \tilde{\sigma}=\left\{\left(\mathrm{u}, \eta_{\widetilde{\sigma}}(\mathrm{u})\right) / \mathrm{u} \in \mathrm{U}\right\} \\
& \tilde{\beta}=\left\{\left(\mathrm{v}, \eta_{\tilde{\beta}}(\mathrm{v})\right) / \mathrm{v} \in \mathrm{V}\right\}
\end{aligned}
$$

Where $\mathrm{X}, \mathrm{Y}, \mathrm{U}$ and $\mathrm{V}$ are crisp universal set of arrival rate, service rate, breakdown rate and repair rate respectively. Let $f(\tilde{\lambda}, \tilde{\mu}, \tilde{\sigma}, \tilde{\beta})$ denote the system characteristic of interest. Since $\tilde{\lambda}, \widetilde{\mu}, \widetilde{\sigma}, \widetilde{\beta}$ are fuzzy numbers $\mathrm{f}(\widetilde{\lambda}, \widetilde{\mu}, \widetilde{\sigma}, \widetilde{\beta})$ is also a fuzzy number.

Following Zadch's extension principle (see $[8,9]$ ), the membership functions of the system characteristic $\mathrm{f}(\tilde{\lambda}, \tilde{\mu}, \widetilde{\sigma}, \widetilde{\beta})$ is defined as

$$
\eta_{\mathrm{f}(\tilde{\lambda}, \tilde{a}, \tilde{\sigma}, \tilde{\beta})}(z)=\sup _{\substack{\mathrm{x} \in \mathrm{X}, \mathrm{y} \in Y \\ u \in U, \mathrm{v} \in V}} \min \left\{\eta_{\chi}(\mathrm{x}), \eta_{\tilde{\mu}}(y), \eta_{\tilde{\sigma}}(\mathrm{u}), \eta_{\tilde{\beta}}(\mathrm{v}) / z=f(\mathrm{x}, \mathrm{y}, \mathrm{u}, \mathrm{v})\right\}
$$

The membership function for the system is idle

$$
\eta_{\mathrm{E}}(\boldsymbol{z})=\sup _{\substack{x \in \mathrm{X}, \mathrm{y} \in \mathrm{Y} \\ \mathrm{u} \in \mathrm{U}, \mathrm{v} \in \mathrm{V}}} \min \left\{\eta_{\tilde{\lambda}}(\mathrm{x}), \eta_{\tilde{\mu}}(\mathrm{y}), \eta_{\widetilde{\sigma}}(\mathrm{u}), \eta_{\tilde{\beta}}(\mathrm{v}) / \mathrm{z}=\frac{\mathrm{vy}-\mathrm{x}(\mathrm{u}+v)}{\mathrm{vy}}\right\}
$$


Likewise, the membership function for the system is idle for type II is

$$
\eta_{\tilde{\mathrm{F}}}(\mathrm{z})=\sup _{\substack{\mathrm{x} \in \mathrm{X}, \mathrm{y} \in \mathrm{Y} \\ \mathrm{u} \in \mathrm{U}, \mathrm{v} \in \mathrm{V}}} \min \left\{\eta_{\tilde{\tilde{\lambda}}}(\mathrm{x}), \eta_{\tilde{\mu}}(\mathrm{y}), \eta_{\tilde{\sigma}}(\mathrm{u}), \eta_{\tilde{\beta}}(\mathrm{v}) / \mathrm{z}=\frac{\mathrm{vy}-\mathrm{x}(\mathrm{u}+\mathrm{v})}{\mathrm{y}(\mathrm{u}+\mathrm{v})}\right\}
$$

In this paper we approach the problem by using mathematical programming, technique. These parametric nonlinear programs are developed to find $\alpha$-cuts of $\mathrm{f}(\tilde{\lambda}, \widetilde{\mu}, \widetilde{\sigma}, \widetilde{\beta})$ by using Zadeh's extension principle.

\section{THE PARAMETRIC NONLINEAR PROGRAMMING APPROACH}

Our approach is to construct the membership functions of $\eta_{\mathrm{f}(\tilde{\lambda}, \tilde{\mu}, \tilde{\sigma}, \tilde{\beta})}$ by deriving the $\alpha$-cuts of $\eta_{\mathrm{f}(\tilde{\lambda}, \not{\mu}, \tilde{\sigma}, \tilde{\beta})}$. The $\alpha$-cuts of $\tilde{\lambda}, \tilde{\mu}, \tilde{\sigma}, \widetilde{\beta}$ are defined as

$$
\begin{aligned}
& \lambda(\alpha)=\left\{\mathrm{x} \in \mathrm{X} / \eta_{\tilde{\lambda}}(\mathrm{x}) \geq \alpha\right\} \\
& \mu(\alpha)=\left\{\mathrm{y} \in \mathrm{Y} / \eta_{\tilde{\mu}}(\mathrm{y}) \geq \alpha\right\} \\
& \sigma(\alpha)=\left\{u \in U / \eta_{\widetilde{\sigma}}(\mathrm{u}) \geq \alpha\right\} \\
& \beta(\alpha)=\left\{\mathrm{v} \in V / \eta_{\tilde{\beta}}(\mathrm{u}) \geq \alpha\right\}
\end{aligned}
$$

The fuzzy arrival rate $\bar{\lambda}$ fuzzy service rate $\bar{\mu}$, fuzzy breakdown rate $\bar{\sigma}$ and fuzzy repair rate $\bar{\beta}$ of $\mathrm{FM} / \mathrm{FM}(\mathrm{FM}, \mathrm{FM}) / \mathrm{I} / \infty$ queuing systems are fuzzy numbers. Therefore the $\alpha$-level sets of $\widetilde{\lambda}, \tilde{\mu}, \widetilde{\sigma}, \widetilde{\beta}$ defined in Equation (5) are crisp intervals which can be expressed in the following forms

$$
\begin{aligned}
& \left.\lambda(\alpha)=\left[\mathrm{x}_{\alpha}^{L}, \mathrm{x}_{\alpha}^{\mathrm{U}}\right]=\min _{\mathrm{x} \in \mathrm{X}}\left\{\mathrm{x} / \eta_{\tilde{\lambda}}(\mathrm{x}) \geq \alpha\right\}, \max _{\mathrm{x} \in \mathrm{X}}\left\{\mathrm{x} / \eta_{\tilde{\lambda}}(\mathrm{x}) \geq \alpha\right\}\right] \\
& \mu(\alpha)=\left[\mathrm{y}_{\alpha}^{L}, \mathrm{x}_{\alpha}^{\mathrm{U}}\right]=\left[\min _{\mathrm{y} \in Y}\left\{\mathrm{y} / \eta_{\tilde{\mu}}(\mathrm{y}) \geq \alpha\right\}, \max _{\mathrm{y} \in Y}\left\{\mathrm{y} / \eta_{\tilde{\mu}}(\mathrm{y}) \geq \alpha\right\}\right] \\
& \left.\sigma(\alpha)=\left[\mathrm{u}_{\alpha}^{L}, \mathrm{u}_{\alpha}^{\mathrm{U}}\right]=\min _{\mathrm{u} \in U}\left\{\mathrm{u} / \eta_{\tilde{\sigma}}(\mathrm{u}) \geq \alpha\right\}, \max _{\mathrm{u} \in U}\left\{\mathrm{u} / \eta_{\tilde{\sigma}}(\mathrm{u}) \geq \alpha\right\}\right] \\
& \left.\beta(\alpha)=\left[\mathrm{v}_{\alpha}^{L}, \mathrm{v}_{\alpha}^{\mathrm{U}}\right]=\min _{\mathrm{v} \in V}\left\{\mathrm{v} / \eta_{\tilde{\beta}}(\mathrm{v}) \geq V\right\}, \max _{\mathrm{v} \in V}\left\{\mathrm{v} / \eta_{\tilde{\beta}}(\mathrm{v}) \geq \mathrm{V}\right\}\right]
\end{aligned}
$$

These intervals indicate where the constant arrival rate, service rate, breakdown rate and repair rate respectively lie at possibility level $\alpha$. We know that $\lambda(\alpha), \mu(\alpha), \sigma(\alpha), \beta(\alpha)$ are crisp sets, rather than fuzzy sets. The FM/FM (FM, FM)/I/ $\infty$ queue can be reduced to a family or crisp $\mathrm{M} / \mathrm{M} \quad(\mathrm{M}, \mathrm{M}) / \mathrm{I} / \infty \quad$ queue with different $\alpha$-level set $\{\lambda(\alpha) / 0<\alpha \leq 1\},\{\mu(\alpha) / 0<\alpha \leq 1\},\{\sigma(\alpha) / 0<\alpha \leq 1\}, \quad\{\beta(\alpha) / 0<\alpha \leq 1\}$. By convexity of a fuzzy number [12], the bounds of these intervals are functions of $\alpha$ and can be obtained as

$x_{\alpha}^{L}=\min \eta_{\tilde{\lambda}}^{-1}(\alpha), \mathrm{x}_{\alpha}^{\mathrm{u}}=\max \eta_{\tilde{\lambda}}^{-1}(\alpha), y_{\alpha}^{L}=\min \eta_{\tilde{\mu}}^{-1}(\alpha), y_{\alpha}^{U}=\max \eta_{\tilde{\mu}}^{-1}(\alpha), u_{\alpha}^{L}=\min \eta_{\tilde{\sigma}}^{-1}(\alpha)$,

$\mathrm{u}_{\alpha}^{\mathrm{u}}=\max \eta_{\tilde{\sigma}}^{-1}(\alpha), \quad \mathrm{v}_{\alpha}^{\mathrm{L}}=\min \eta_{\mathfrak{\beta}}^{-1}(\alpha), \mathrm{v}_{\alpha}^{\mathrm{U}}=\max \eta_{\mathfrak{\beta}}^{-1}(\alpha)$ respectively.

Consider the membership function of the expected time that the system is idle in Type I system

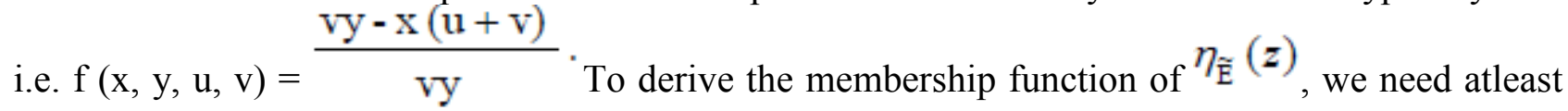
one of the following cases held such that 
$\mathrm{Z}=\frac{v y-x(u+v)}{v y}$ to satisfy $\eta_{\mathrm{E}}(z)=\alpha$.

Case (i) : $\left(\eta_{\tilde{\lambda}}(\mathrm{x})=\alpha, \eta_{\tilde{\mu}}(\mathrm{y}) \geq \alpha, \eta_{\tilde{\sigma}}(\mathrm{u}) \geq \alpha, \eta_{\tilde{\beta}}(\mathrm{v}) \geq \alpha\right)$,

Case (ii) : $\left(\eta_{\tilde{\lambda}}(\mathrm{x}) \geq \alpha, \eta_{\tilde{\mu}}(\mathrm{y})=\alpha, \eta_{\widetilde{\gamma}}(\mathrm{u}) \geq \alpha, \eta_{\tilde{\beta}}(\mathrm{v}) \geq \alpha\right)$,

Case (iii) : $\left(\eta_{\tilde{\lambda}}(\mathrm{x})=\alpha, \eta_{\tilde{\mu}}(\mathrm{y}) \geq \alpha, \eta_{\tilde{\sigma}}(\mathrm{u})=\alpha, \eta_{\tilde{\beta}}(\mathrm{v}) \geq \alpha\right)$,

Case (iv) : $\left(\eta_{\widetilde{\lambda}}(\mathrm{x}) \geq \alpha, \eta_{\tilde{a}}(\mathrm{y}) \geq \alpha, \eta_{\widetilde{\sigma}}(\mathrm{u}) \geq \alpha, \eta_{\widetilde{\gamma}}(\mathrm{v})=\alpha\right)$

These can be accomplished by parametric NLP techniques. For case (i), the corresponding parametric nonlinear programmes for finding lower and upper bounds of the $\alpha$-cut of $\tilde{I}$ are

$$
\mathrm{E}_{\alpha}^{\mathrm{L}_{1}}=\min _{\mathrm{x}, \mathrm{y}, \mathrm{u}, \mathrm{v} \in \mathrm{R}^{+}} \frac{\mathrm{vy}-x(u+v)}{v y}
$$

Satisfy $x_{\alpha}^{\mathrm{L}} \leq \mathrm{x} \leq \mathrm{x}_{\alpha}^{\mathrm{U}}, \mathrm{y} \in \mu(\alpha), \mathrm{u} \in \sigma(\alpha)$ and $\mathrm{v} \in \beta(\alpha)$

$$
\mathrm{E}_{\alpha}^{\mathrm{U}_{1}}=\max _{\mathrm{x}, \mathrm{y}, \mathrm{u}, \mathrm{v} \in \mathrm{R}^{+}} \frac{\mathrm{vy}-x(u+v)}{v y}
$$

Satisfy $x_{\alpha}^{\mathrm{L}} \leq \mathrm{x} \leq \mathrm{x}_{\alpha}^{\mathrm{U}}, \mathrm{y} \in \mu(\alpha), \mathrm{u} \in \sigma(\alpha)$ and $\mathrm{v} \in \beta(\alpha)$

For case (ii) are :

$$
\mathrm{E}_{\alpha}^{\mathrm{L}_{2}}=\min _{\mathrm{x}, \mathrm{y}, \mathrm{u}, \mathrm{v} \in \mathrm{R}^{+}} \frac{\mathrm{vy}-x(u+v)}{v y}
$$

Satisfy $x \in \lambda(\alpha), \mathrm{y}_{\alpha}^{\mathrm{L}} \leq \mathrm{y} \leq \mathrm{y}_{\alpha}^{\mathrm{U}}, \mathrm{u} \in \sigma(\alpha)$ and $\mathrm{v} \in \beta(\alpha)$

$$
\mathrm{E}_{\alpha}^{\mathrm{U}_{2}}=\max _{\mathrm{x}, \mathrm{y}, \mathrm{u}, \mathrm{v} \in \mathrm{R}^{+}} \frac{\mathrm{vy}-x(u+v)}{v y}
$$

Satisfy $x \in \lambda(\alpha), \mathrm{y}_{\alpha}^{\mathrm{L}} \leq \mathrm{y} \leq \mathrm{y}_{\alpha}^{\mathrm{U}}, \mathrm{u} \in \sigma(\alpha)$ and $\mathrm{v} \in \beta(\alpha)$

For case (iii) are

$$
\mathrm{E}_{\alpha}^{\mathrm{L}_{3}}=\min _{\mathrm{x}, \mathrm{y}, \mathrm{u}, \mathrm{v} \in \mathbb{R}^{+}} \frac{\mathrm{vy}-x(u+v)}{v y}
$$

Satisfy $x \in \lambda(\alpha), \mathrm{y} \in \mu(\alpha), \mathrm{u}_{\alpha}^{\mathrm{L}} \leq \mathrm{u} \leq \mathrm{u}_{\alpha}^{\mathrm{U}}$ and $\mathrm{v} \in \beta(\alpha)$

$$
\mathrm{E}_{\alpha}^{\mathrm{U}_{3}}=\max _{\mathrm{x}, \mathrm{y}, \mathrm{u}, \mathrm{v} \in \mathrm{R}^{+}} \frac{\mathrm{vy}-x(u+v)}{v y}
$$

Satisfy $x \in \lambda(\alpha), \mathrm{y} \in \mu(\alpha), \mathrm{u}_{\alpha}^{\mathrm{L}} \leq \mathrm{u} \leq \mathrm{u}_{\alpha}^{\mathrm{U}}$ and $\mathrm{v} \in \beta(\alpha)$

For case (iv) are

$$
\mathrm{E}_{\alpha}^{\mathrm{L}_{4}}=\min _{\mathrm{x}, \mathrm{y}, \mathrm{u}, \mathrm{v} \in \mathrm{R}^{+}} \frac{\mathrm{vy}-x(u+v)}{v y}
$$

Satisfy $x \in \lambda(\alpha), \mathrm{y} \in \mu(\alpha), \mathrm{u} \in \sigma(\alpha), \mathrm{u}_{\alpha}^{\mathrm{L}} \leq u \leq v_{\alpha}^{\mathrm{u}}$ 


$$
\mathrm{E}_{\alpha}^{\mathrm{U}_{4}}=\max _{\mathrm{x}, \mathrm{y}, \mathrm{u}, \mathrm{v} \in \mathrm{R}^{+}} \frac{\mathrm{vy}-x(u+v)}{v y}
$$

Satisfy $x \in \lambda(\alpha), \mathrm{y} \in \mu(\alpha), \mathrm{u} \in \sigma(\alpha), \mathrm{v}_{\alpha}^{\mathrm{L}} \leq v \leq u_{\alpha}^{\mathrm{u}}$

From the definition of $\lambda(\alpha), \mu(\alpha), \sigma(\alpha), \beta(\alpha)$ in Eqn. (6), $x \in \lambda(\alpha)$, $\mathrm{y} \in \mu(\alpha), \mathrm{u} \in \sigma(\alpha)$ and $\mathrm{v} \in \beta(\alpha)$ can be replaced by $\mathrm{x} \in\left[x_{\alpha}^{\mathrm{L}}, \mathrm{x}_{\alpha}^{\mathrm{U}}\right], \mathrm{y} \in\left\lfloor y_{\alpha}^{\mathrm{L}}, \mathrm{y}_{\alpha}^{\mathrm{U}}\right\rfloor$, $\mathrm{u} \in\left[u_{\alpha}^{\mathrm{L}}, \mathrm{u}_{\alpha}^{\mathrm{U}}\right]$ and $\mathrm{v} \in\left\lfloor v_{\alpha}^{\mathrm{L}}, \mathrm{v}_{\alpha}^{\mathrm{U}}\right\rfloor$ respectively. All $\alpha$-cuts form a nested structure with respect to $\alpha$ (See $[5,12])$ ie. Given $0<\alpha_{2}<\alpha_{1} \leq 1$, we've $\left[\mathrm{x}_{\alpha_{1}}^{L}, \mathrm{x}_{\alpha_{1}}^{U}\right] \subseteq\left\lfloor\mathrm{x}_{\alpha_{2}}^{L}, \mathrm{x}_{\alpha_{2}}^{U}\right\rfloor$, $\left[y_{\alpha_{1}}^{L}, \mathrm{y}_{\alpha_{1}}^{U}\right] \subseteq\left[\mathrm{y}_{\alpha_{2}}^{\mathrm{L}}, \mathrm{y}_{\alpha_{2}}^{U}\right],\left[\mathrm{u}_{\alpha_{1}}^{L}, u_{\alpha_{1}}^{U}\right] \subseteq\left[\mathrm{u}_{\alpha_{2}}^{L}, \mathrm{u}_{\alpha_{2}}^{U}\right]$ and $\left[\mathrm{v}_{\alpha_{1}}^{L}, \mathrm{v}_{\alpha_{1}}^{U}\right] \subseteq\left[\mathrm{v}_{\alpha_{2}}^{L}, \mathrm{v}_{\alpha_{2}}^{U}\right] \cdot$ Therefore $7(\mathrm{c}),(7 \mathrm{e})$ and $(7 \mathrm{~g})$ have the smallest element and (7b), (7d), (7f) and (7h) have the largest element. To find the membership function $\eta_{\tilde{\mathrm{E}}}$, it is enough to find the left shape function and the right shape function of $\eta_{\tilde{\mathrm{E}}}$, which is equivalent to find the lower bound $\mathrm{E}_{\alpha}^{\mathrm{L}}$ and upper bound $\mathrm{E}_{\alpha}^{\mathrm{U}}$ of the $\alpha$-cuts of $\tilde{i}$, which based on Eq. (4) can be rewritten as

$$
\mathrm{E}_{\alpha}^{\mathrm{L}}=\min _{\mathrm{x}, \mathrm{y}, \mathrm{u}, \mathrm{v} \in \mathrm{R}+} \frac{\mathrm{vy}-x(\mathrm{u}+\mathrm{v})}{v y}
$$

Satisfy $x_{\alpha}^{\mathrm{L}} \leq x \leq \mathrm{x}_{\alpha}^{\mathrm{U}}, \mathrm{y}_{\alpha}^{\mathrm{L}} \leq y \leq \mathrm{y}_{\alpha}^{\mathrm{U}}, \mathrm{u}_{\alpha}^{\mathrm{L}} \leq u \leq u_{\alpha}^{\mathrm{U}}, \mathrm{v}_{\alpha}^{\mathrm{L}} \leq v \leq v_{\alpha}^{\mathrm{U}}$

$$
\mathrm{E}_{\alpha}^{\mathrm{U}}=\max _{\mathrm{x}, \mathrm{y}, \mathrm{u}, \mathrm{v} \in \mathrm{R}+} \frac{\mathrm{vy}-x(\mathrm{u}+\mathrm{v})}{v y}
$$

Satisfy $x_{\alpha}^{\mathrm{L}} \leq x \leq x_{\alpha}^{\mathrm{U}}, \mathrm{y}_{\alpha}^{\mathrm{L}} \leq y \leq y_{\alpha}^{\mathrm{U}}, \mathrm{u}_{\alpha}^{\mathrm{L}} \leq u \leq u_{\alpha}^{\mathrm{U}}, \mathrm{v}_{\alpha}^{\mathrm{L}} \leq v \leq \mathrm{v}_{\alpha}^{\mathrm{U}}$

Atleast one $\mathrm{x}, \mathrm{y}, \mathrm{u}$ or $\mathrm{v}$ must hit the boundaries of their $\alpha$-cuts to satisfy $\eta_{\tilde{\mathrm{E}}}(z)=\alpha$.

This model is a pair of mathematical programs with bound constraints, which involves the systematic study of how the optional solutions change when $x_{\alpha}^{\mathrm{L}}, x_{\alpha}^{\mathrm{U}}, \mathrm{y}_{\alpha}^{\mathrm{L}}, \mathrm{y}_{\alpha}^{\mathrm{U}}, u_{\alpha}^{\mathrm{L}}, u_{\alpha}^{\mathrm{U}}, v_{\alpha}^{\mathrm{L}}, v_{\alpha}^{\mathrm{U}}$ vary over the interval $\alpha \in(0,1]$. They fall into category of parametric NLP (2).

The crisp interval $\left\lfloor E_{\alpha}^{L}, E_{\alpha}^{U}\right\rfloor$ solved from Eq. (8) represents the $\alpha$-cuts of $\tilde{I}$. Again, by applying the results by Zimmermann [10] and Kaufmann [11] and the convexity properties of $\tilde{i}$, we have $E_{\alpha_{1}}^{\mathrm{L}} \geq \mathrm{E}_{\alpha_{2}}^{L}$ and $\mathrm{E}_{\alpha_{1}}^{\mathrm{U}} \leq \mathrm{E}_{\alpha_{2}}^{U}$ where $0<\alpha_{1}<\alpha_{2}<\leq \leq 1$. In other words, $\mathrm{E}_{\alpha}^{\mathrm{L}}$ is increasing with respect to $\alpha$ and $E_{\alpha}^{U}$ is decreasing with respect $\alpha$. Consequently the membership function $\eta_{\tilde{\mathrm{E}}}(z)$ can be found from Eq. (8).

If both $E_{\alpha}^{\mathrm{L}}$ and $\mathrm{E}_{\alpha}^{\mathrm{U}}$ are invertible with respect to $\alpha$, then a left shape function $\mathrm{L}(\mathrm{z})=\left(\mathrm{E}_{\alpha}{ }^{\mathrm{L}}\right)^{-1}$ and a right shape function $\mathrm{R}(\mathrm{z})=\left(E_{\alpha}{ }^{U}\right)^{-1}$ can be obtained, from which the membership function $\eta_{\tilde{\mathrm{E}}}$ is constructed.

$$
\eta_{\tilde{\mathrm{E}}}(z)=\left\{\begin{array}{l}
\mathrm{L}(\mathrm{z}), \mathrm{z}_{1} \leq z \leq z_{2} \\
1, \quad \mathrm{z}_{2} \leq z \leq z_{3} \\
\mathrm{R}(\mathrm{z}), \mathrm{z}_{3} \leq z \leq z_{4}
\end{array}\right.
$$


In most cases, the values of $z_{i}$ cannot be solved analytically. Consequently, a closed-form membership function for $\tilde{I}$ cannot be obtained. In the following section, we will present an efficient solution algorithm to compute the membership values (ie. $E_{\alpha}^{\mathrm{L}}, \mathrm{E}_{\alpha}^{\mathrm{U}}$ ) of system characteristic at a different possibility level $\alpha$.

The membership function for $\tilde{I}$, the expected time that the system is idle in Type II system can be derived in a similar manner.

\section{Solution algorithm}

Let the arrival rate, service rate, breakdown rate and repair rate be trapezoidal fuzzy number represented by $\left[\mathrm{x}_{1}, \mathrm{x}_{2}, \mathrm{x}_{3}, \mathrm{x}_{4}\right] . \quad\left[\mathrm{y}_{1}, \mathrm{y}_{2}, \mathrm{y}_{3}, \mathrm{y}_{4}\right], \quad\left[\mathrm{u}_{1}, \mathrm{u}_{2}, \mathrm{u}_{3}, \mathrm{u}_{4}\right]$ and $\left[\mathrm{v}_{1}, \mathrm{v}_{2}, \mathrm{v}_{3}, \mathrm{v}_{4}\right]$ per unit time respectively, where $\mathrm{x}_{4}>\mathrm{x}_{3}>\mathrm{x}_{2}>\mathrm{x}_{1}, \quad \mathrm{y}_{4}>\mathrm{y}_{3}>\mathrm{y}_{2}>\mathrm{y}_{1}, \quad \mathrm{u}_{4}>\mathrm{u}_{3}>\mathrm{u}_{2}>\mathrm{u}_{1}, \quad \mathrm{v}_{4}>\mathrm{v}_{3}>\mathrm{v}_{2}>\mathrm{v}_{1}$. Then we have the membership functions $\widetilde{\lambda}, \tilde{\mu}, \widetilde{\sigma}, \widetilde{\beta}$

$$
\begin{gathered}
\eta_{\tilde{\lambda}}(x)= \begin{cases}\left(\mathrm{x}-\mathrm{x}_{1}\right) /\left(\mathrm{x}_{2}-x_{1}\right) & \mathrm{x}_{1} \leq x \leq x_{2} \\
1 & \mathrm{x}_{2} \leq \mathrm{x} \leq x_{3} \\
\left(\mathrm{x}_{4}-x\right) /\left(\mathrm{x}_{4}-x_{3}\right) & \mathrm{x}_{3} \leq x \leq x_{4}\end{cases} \\
\eta_{\tilde{\mu}}(y)= \begin{cases}\left(\mathrm{y}-\mathrm{y}_{1}\right) /\left(\mathrm{y}_{2}-y_{1}\right) & \mathrm{y}_{1} \leq y \leq y_{2} \\
1 & \mathrm{y}_{2} \leq \mathrm{y} \leq y_{3} \\
\left(\mathrm{y}_{4}-y\right) /\left(\mathrm{y}_{4}-y_{3}\right) & \mathrm{y}_{3} \leq y \leq y_{4}\end{cases} \\
\eta_{\widetilde{\sigma}}(u)= \begin{cases}\left(\mathrm{u}-\mathrm{u}_{1}\right) /\left(\mathrm{u}_{2}-u_{1}\right) & \mathrm{u}_{1} \leq u \leq u_{2} \\
1 & \mathrm{u}_{2} \leq \mathrm{u} \leq u_{3} \\
\left(\mathrm{u}_{4}-v\right) /\left(\mathrm{u}_{4}-u_{3}\right) & \mathrm{u}_{3} \leq u \leq u_{4}\end{cases} \\
\eta_{\tilde{\beta}}(v)= \begin{cases}\left(\mathrm{v}-\mathrm{v}_{1}\right) /\left(\mathrm{v}_{2}-v_{1}\right) & \mathrm{v}_{1} \leq v \leq v_{2} \\
1 & \mathrm{v}_{2} \leq \mathrm{v} \leq v_{3} \\
\left(\mathrm{v}_{4}-v\right) /\left(\mathrm{v}_{4}-v_{3}\right) & \mathrm{v}_{3} \leq v \leq v_{4}\end{cases}
\end{gathered}
$$

The system manager wants to evaluate the system characteristic such as the expected time that the system is idle. Using the proposed approach stated in section 3, it is easy to obtain the $\alpha$ cuts of $\tilde{\lambda}, \tilde{\mu}, \tilde{\sigma}, \widetilde{\beta}$ as follows :

$$
\begin{aligned}
& {\left[\mathrm{x}_{\alpha}^{\mathrm{L}}, \mathrm{x}_{\alpha}^{\mathrm{U}}\right]=\left[\min ^{-1} \eta_{\tilde{\lambda}}(\alpha), \max ^{-1} \eta_{\tilde{\lambda}}(\alpha)\right]=\left[\left(\mathrm{x}_{2}-x_{1}\right) \alpha+\mathrm{x}_{1}, \mathrm{x}_{4}-\left(x_{4}-x_{3}\right) \alpha\right]} \\
& {\left[\mathrm{y}_{\alpha}^{\mathrm{L}}, \mathrm{y}_{\alpha}^{\mathrm{U}}\right]=\left[\min ^{-1} \eta_{\tilde{\mu}}(\alpha), \max ^{-1} \eta_{\tilde{\mu}}(\alpha)\right]=\left[\left(\mathrm{y}_{2}-y_{1}\right) \alpha+\mathrm{y}_{1}, \mathrm{y}_{4}-\left(y_{4}-y_{3}\right) \alpha\right]} \\
& {\left[\mathrm{u}_{\alpha}^{\mathrm{L}}, \mathrm{u}_{\alpha}^{\mathrm{U}}\right]=\left[\min ^{-1} \eta_{\widetilde{\sigma}}(\alpha), \max ^{-1} \eta_{\widetilde{\gamma}}(\alpha)\right]=\left[\left(\mathrm{u}_{2}-u_{1}\right) \alpha+\mathrm{u}_{1}, \mathrm{u}_{4}-\left(u_{4}-u_{3}\right) \alpha\right]}
\end{aligned}
$$

And

$$
\left[\mathrm{v}_{\alpha}^{\mathrm{L}}, \mathrm{v}_{\alpha}^{\mathrm{U}}\right]=\left[\min ^{-1} \eta_{\tilde{\beta}}(\alpha), \max ^{-1} \eta_{\tilde{\beta}}(\alpha)\right]=\left[\left(\mathrm{v}_{2}-v_{1}\right) \alpha+\mathrm{v}_{1}, \mathrm{v}_{4}-\left(v_{4}-v_{3}\right) \alpha\right]
$$

The following (8), a set of parametric nonlinear programs for deriving the membership function of $\mathrm{E}$ for Type I - FM/FM(FM,FM/1/o system are given by

$$
\mathrm{E}_{\alpha}^{\mathrm{L}}=\min _{\mathrm{x}, \mathrm{y}, \mathrm{u}, \mathrm{v} \in \mathrm{R}^{+}} \frac{\mathrm{vy}-x(u+v)}{\mathrm{vy}}
$$

Satisfy $\left(\mathrm{x}_{2}-\mathrm{x}_{1}\right) \alpha+\mathrm{x}_{1} \leq \mathrm{x} \leq \mathrm{x}_{4}-\left(\mathrm{x}_{4}-\mathrm{x}_{3}\right) \alpha$,

$$
\left(\mathrm{y}_{2}-\mathrm{y}_{1}\right) \alpha+\mathrm{y}_{1} \leq \mathrm{y} \leq \mathrm{y}_{4}-\left(\mathrm{y}_{4}-\mathrm{y}_{3}\right) \alpha
$$




$$
\begin{aligned}
& \left(\mathrm{u}_{2}-\mathrm{u}_{1}\right) \alpha+\mathrm{u}_{1} \leq \mathrm{u} \leq \mathrm{u}_{4}-\left(\mathrm{u}_{4}-\mathrm{u}_{3}\right) \alpha, \\
& \left(\mathrm{v}_{2}-\mathrm{v}_{1}\right) \alpha+\mathrm{v}_{1} \leq \mathrm{v} \leq \mathrm{v}_{4}-\left(\mathrm{v}_{4}-\mathrm{v}_{3}\right) \alpha, \\
E_{\alpha}^{\mathrm{U}}= & \max _{\mathrm{x}, \mathrm{y}, \mathrm{u}, \mathrm{v} \in \mathrm{R}^{+}} \frac{\mathrm{vy}-\mathrm{x}(\mathrm{u}+\mathrm{v})}{v y}
\end{aligned}
$$

Satisfy $\left(\mathrm{x}_{2}-\mathrm{x}_{1}\right) \alpha+\mathrm{x}_{1} \leq \mathrm{x} \leq \mathrm{x}_{4}-\left(\mathrm{x}_{4}-\mathrm{x}_{3}\right) \alpha$,

$$
\begin{aligned}
& \left(\mathrm{y}_{2}-\mathrm{y}_{1}\right) \alpha+\mathrm{y}_{1} \leq \mathrm{y} \leq \mathrm{y}_{4}-\left(\mathrm{y}_{4}-\mathrm{y}_{3}\right) \alpha, \\
& \left(\mathrm{u}_{2}-\mathrm{u}_{1}\right) \alpha+\mathrm{u}_{1} \leq \mathrm{u} \leq \mathrm{u}_{4}-\left(\mathrm{u}_{4}-\mathrm{u}_{3}\right) \alpha, \\
& \left(\mathrm{v}_{2}-\mathrm{v}_{1}\right) \alpha+\mathrm{v}_{1} \leq \mathrm{v} \leq \mathrm{v}_{4}-\left(\mathrm{v}_{4}-\mathrm{v}_{3}\right) \alpha,
\end{aligned}
$$

Note that the parametric nonlinear programs for deriving the membership function of $\mathrm{F}$ for Type

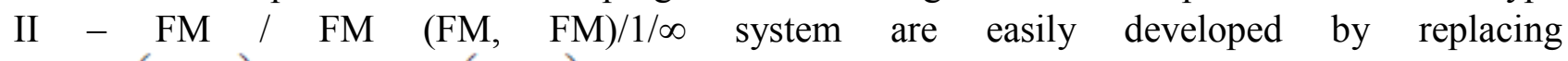
$\frac{v y-x(\mathrm{u}+\mathrm{v})}{\mathrm{vy}}$ by $\frac{\mathrm{vy}-x(\mathrm{u}+\mathrm{v})}{\mathrm{y}(\mathrm{u}+\mathrm{v})}$

We can summarize the solution procedure into the following algorithm.

Input the arrival rate, service rate, breakdown rate and repair rate which are trapezoidal fuzzy number represented by $\left(\mathrm{x}_{1}, \mathrm{x}_{2}, \mathrm{x}_{3}, \mathrm{x}_{4}\right),\left(\mathrm{y}_{1}, \mathrm{y}_{2}, \mathrm{y}_{3}, \mathrm{y}_{4}\right),\left(\mathrm{u}_{1}, \mathrm{u}_{2}, \mathrm{u}_{3}, \mathrm{u}_{4}\right),\left(\mathrm{v}_{1}, \mathrm{v}_{2}, \mathrm{v}_{3}, \mathrm{v}_{4}\right)$.

Output the numbers $\mathrm{x}_{\alpha}^{\mathrm{L}}, \mathrm{x}_{\alpha}^{\mathrm{U}}, \mathrm{y}_{\alpha}^{\mathrm{L}}, \mathrm{y}_{\alpha}^{\mathrm{U}}, \mathrm{u}_{\alpha}^{\mathrm{L}}, \mathrm{u}_{\alpha}^{\mathrm{U}}, \mathrm{v}_{\alpha}^{\mathrm{L}}, v_{\alpha}^{\mathrm{U}}, \mathrm{f}_{\alpha}^{\mathrm{L}}, \mathrm{f}_{\alpha}^{\mathrm{U}}$

Step 1 for $\alpha=0$ to 1 steps $\Delta$

Step 2

$$
\begin{aligned}
& \mathrm{x}_{\alpha}^{\mathrm{L}}=\left(\mathrm{x}_{2}-x_{1}\right) \alpha+\mathrm{x}_{1} ; \mathrm{x}_{\alpha}^{\mathrm{U}}=x_{4}-\left(x_{4}-x_{3}\right) \alpha ; \\
& \mathrm{y}_{\alpha}^{\mathrm{L}}=\left(\mathrm{y}_{2}-y_{1}\right) \alpha+\mathrm{y}_{1} ; \mathrm{y}_{\alpha}^{\mathrm{U}}=y_{4}-\left(y_{4}-y_{3}\right) \alpha ; \\
& \mathrm{u}_{\alpha}^{\mathrm{L}}=\left(\mathrm{u}_{2}-u_{1}\right) \alpha+\mathrm{u}_{1} ; \mathrm{u}_{\alpha}^{\mathrm{U}}=u_{4}-\left(u_{4}-u_{3}\right) \alpha ; \\
& \mathrm{v}_{\alpha}^{\mathrm{L}}=\left(\mathrm{v}_{2}-v_{1}\right) \alpha+\mathrm{v}_{1} ; \mathrm{v}_{\alpha}^{\mathrm{U}}=v_{4}-\left(v_{4}-v_{3}\right) \alpha ;
\end{aligned}
$$

Step 3 for $\mathrm{x}=x_{\alpha}^{\mathrm{L}}$ to $\mathrm{x}_{\alpha}^{U}$;

Step 4 for $\mathrm{y}=y_{\alpha}^{\mathrm{L}}$ to $\mathrm{y}_{\alpha}^{U}$;

Step 5 for $\mathrm{u}=u_{\alpha}^{\mathrm{L}}$ to $\mathrm{u}_{\alpha}^{U}$;

Step 6 for $v=v_{\alpha}^{\mathrm{L}}$ to $\mathrm{v}_{\alpha}^{\mathrm{U}}$;

Step $7 \mathrm{f}_{\alpha}^{\mathrm{L}}=\arg \{\min \mathrm{f}(\mathrm{x}, \mathrm{y}, \mathrm{u}, \mathrm{v})\} ; \mathrm{f}_{\alpha}^{\mathrm{U}}=\arg \{\max \mathrm{f}(\mathrm{x}, \mathrm{y}, \mathrm{u}, \mathrm{v})\}$

Step 8 output $x_{\alpha}^{\mathrm{L}}, \mathrm{x}_{\alpha}^{\mathrm{U}}, \mathrm{y}_{\alpha}^{\mathrm{L}}, \mathrm{y}_{\alpha}^{\mathrm{U}}, \mathrm{u}_{\alpha}^{\mathrm{L}}, \mathrm{u}_{\alpha}^{\mathrm{U}}, \mathrm{v}_{\alpha}^{\mathrm{L}}, \mathrm{v}_{\alpha}^{\mathrm{U}}, \mathrm{f}_{\alpha}^{\mathrm{L}}, \mathrm{f}_{\alpha}^{\mathrm{U}}$.

Stop. 
It should be noted that the numerical solutions of $\mathrm{f}_{\alpha}^{\mathrm{L}}$ and $\mathrm{f}_{\alpha}^{\mathrm{U}}$ at different $\alpha$ levels can be generated to approximate the shape of $\mathrm{L}(\mathrm{z})$ and $\mathrm{R}(\mathrm{z})$, from which the membership function can be constructed.

\section{NUMERICAL EXAMPLES}

To demonstrate how the proposed approach can be applied to analyse fuzzy queue with an reliable server, we present some examples often encountered in a real-world fuzzy environment. Due to the complexity of four fuzzy variable, it is impossible to determine the analytical solution of crisp interval $\left[E_{\alpha}^{\mathrm{L}}, \mathrm{E}_{\alpha}^{\mathrm{U}}\right]$ interms of $\alpha$. Consequently, it is very difficult to obtain the closed-form membership function of $\eta_{\tilde{\mathrm{E}}}$. Instead, a software Matlab 6.0 for windows is used to solve the mathematical programmes and then the shape of $\eta \mathrm{E}$ can be found for a given $\alpha$. Here we enumerate $11 \alpha$ values $\alpha: 0,0.1,0.2 \ldots .1 .0$. The figures depicts the rough shape of $\eta_{\tilde{\mathrm{E}}}$ from these $11 \alpha$ values.

\section{Example 1}

Consider a load/unload system in which trucks using or single-channel loading dock arrive according to a Poisson process. The time required to load / unload process may be interrupted following a Poisson process. The recovery times of the load / unload interrupted follows an exponential distribution. The loading / unloading resumes as soon as the interruption ends. The arrival rate, service rate, breakdown rate and repair rate are trapezoidal fuzzy numbers represented by $[2,4,5,7],[12,14,16,20],[.05,0.1,0.2,0.5],[2,3,5,6]$ per hour. In attempting to evaluate the single-channel loading dock, the manager of the system wishes to evaluate how many hours that the system is idle. It is evident that this system follows Type I, FM/FM(FM,FM)/1/ $\infty$ and the expected time that the system is idle can be derived by the proposed procedure. Numerical solutions for different values of $\alpha$ are performed to get the crisp intervals $\left[\mathrm{E}_{\alpha}^{\mathrm{L}}, \mathrm{E}_{\alpha}^{\mathrm{U}}\right]$ which approximate the corresponding membership function displayed in fig-I. The rough shape turns out to be quite well and it looks like a continuous function. The $\alpha$-cuts of arrival rates, service rates, breakdown rates and repair rates and the expected time that the system is idle $(\tilde{I})$ are obtained at different possibility levels which are depicted in Table-I. At one extreme end for possibility level $\alpha=1$, the range of fuzzy expected hours that the dock is idle is approximately [0.6190, 0.7450], which indicates that it is definitely possible that the expected time the dock is idle fall between 0.6190 and 0.7450 . At the other end for the possibility level $\alpha=0$, the range of the fuzzy expected time that the dock is idle is approximately [0.2708, 0.8992]. This range indicates that the expected time that the dock is idle will never exceed 0.8992 (or) full below 0.2708 The above result will be certainly useful and significant for the system designers. 
Table 1

System performance measure of expected idle time

\begin{tabular}{|c|c|c|c|c|c|c|c|c|c|c|}
\hline$\alpha$ & $\mathbf{x 1}$ & $\mathbf{x u}$ & $\mathbf{y l}$ & $\mathbf{y u}$ & $\mathbf{u l}$ & $\mathbf{u u}$ & $\mathbf{v 1}$ & $\mathbf{v u}$ & el & eu \\
\hline 0.00 & 2.00 & 7.00 & 12.00 & 20.00 & 0.05 & 0.50 & 2.00 & 6.00 & 0.2708 & 0.8992 \\
\hline 0.10 & 2.20 & 6.80 & 12.20 & 19.60 & 0.0 & 0.47 & 2.10 & 5.90 & 0.3179 & 0.8867 \\
\hline 0.20 & 2.40 & 6.60 & 12.40 & 19.20 & 0.06 & 0.44 & 2.20 & 5.80 & 0.3613 & 0.8737 \\
\hline 0.30 & 2.60 & 6.40 & 12.60 & 18.80 & 0.07 & 0.41 & 2.30 & 5.70 & 0.4015 & 0.8601 \\
\hline 0.40 & 2.80 & 6.20 & 12.80 & 18.40 & 0.07 & 0.38 & 2.40 & 5.60 & 0.4389 & 0.8459 \\
\hline 0.50 & 3.00 & 6.00 & 13.00 & 18.00 & 0.08 & 0.35 & 2.50 & 5.50 & 0.4738 & 0.8311 \\
\hline 0.60 & 3.20 & 5.80 & 13.20 & 17.60 & 0.08 & 0.32 & 2.60 & 5.40 & 0.5065 & 0.8155 \\
\hline 0.70 & 3.40 & 5.60 & 13.40 & 17.20 & 0.09 & 0.29 & 2.70 & 5.30 & 0.5372 & 0.7992 \\
\hline 0.80 & 3.60 & 5.40 & 13.60 & 16.80 & 0.09 & 0.26 & 2.80 & 5.20 & 0.5611 & 0.7820 \\
\hline 0.90 & 3.80 & 5.20 & 13.80 & 16.40 & 0.10 & 0.23 & 2.90 & 5.10 & 0.5933 & 0.7640 \\
\hline 1.00 & 4.00 & 5.00 & 14.00 & 16.00 & 0.10 & 0.20 & 3.00 & 5.00 & 0.6190 & 0.7450 \\
\hline
\end{tabular}

System performance measurement

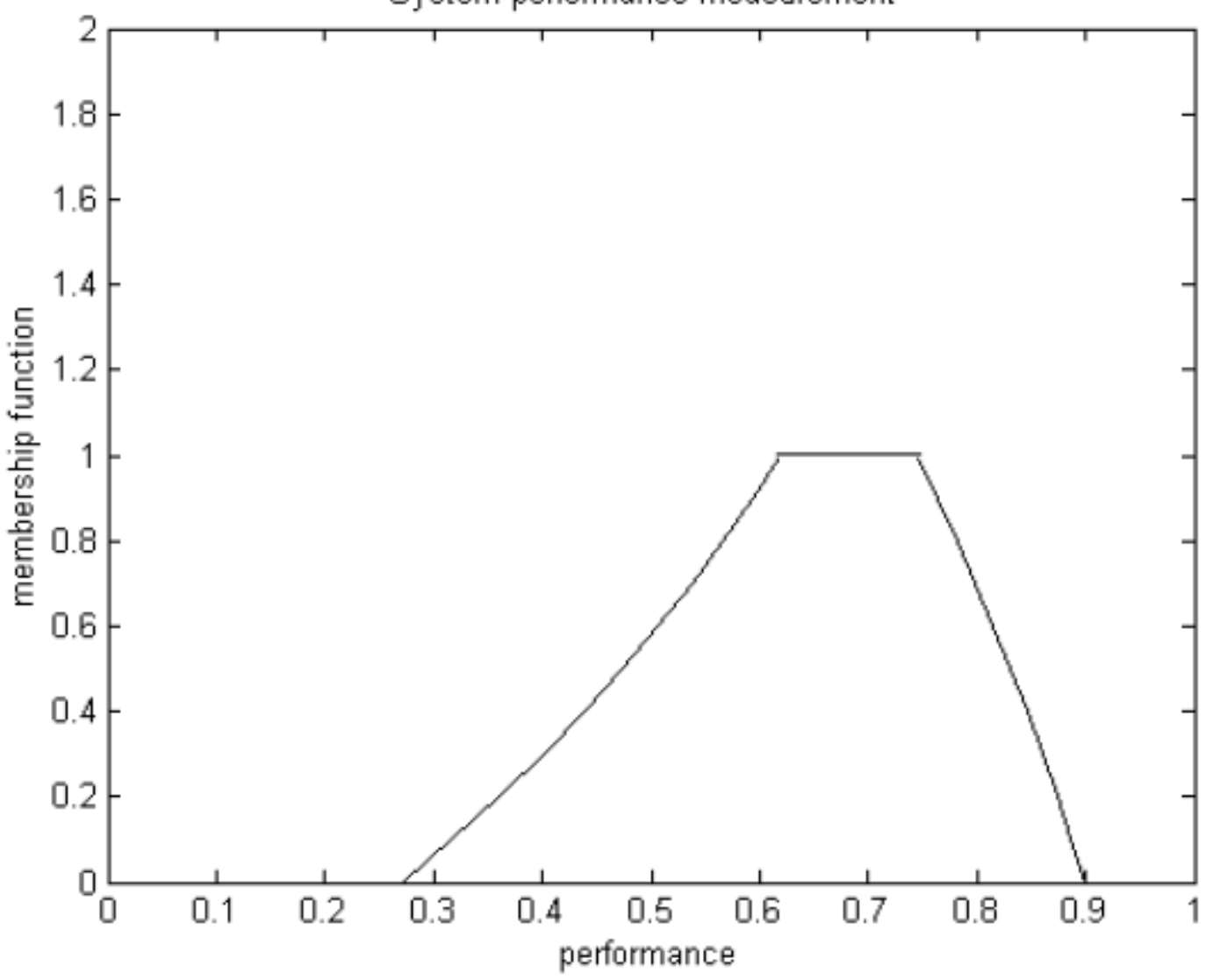

Fig. 1.

The $\alpha$-cuts of arrival rates, service rates, breakdown rates, repair rates and the expected hours that the system is idle is in Example I. 


\section{Example 2}

Consider a parallel processing system where jobs arrive according to a Poisson process, the processing times have an exponential distribution, the service may be interrupted follows Poisson and the recovery times follows an exponential distribution. The system can handle only one job at a time-and the system resumes as soon as the interruption ends. The arrival rate, service rate, breakdown rate and repair rate are fuzzy numbers represented by [10, 20, 30, 40], [100, 200, 300, 500]. [0.01, 0.02, 0.04, 0.05], [1,3,8,10] per hour respectively. It is evident that this system falls in Type I - FM / FM (FM, FM) / $1 / \infty$.

The manager of the system wants to know the system characteristic such as how many hours the processing system is idle. By continuing in a similar manner explained in Example 1, we have fig. 2 and table 2 depicting the approximate membership function for $\tilde{i}$ and the $\alpha$-cuts of the arrival rates, services, breakdown rates, repair rates and the expected time that the system is idle. At one extreme and at possibility level $\alpha=1$, the range of fuzzy expected time that the system is idle is approximately [0.8480 .9332], implying that it is definitely possible that the system time is idle falls between 0.8480 and 0.9332 . On the other and at possibility level $\alpha=0$, the fuzzy expected time that the system is idle is approximately $[0.5800,0.9800]$ which indicates that the expected time the system is idle will never exceed 0.9800 (or) fall below 0.5800 .

Table 2

System performance measure of expected idle time

\begin{tabular}{|c|c|c|c|c|c|c|c|c|c|c|}
\hline$\alpha$ & $\mathbf{x} \mathbf{1}$ & $\mathbf{x u}$ & $\mathbf{y l}$ & $\mathbf{y u}$ & $\mathbf{u l}$ & $\mathbf{u u}$ & $\mathbf{v 1}$ & $\mathbf{v u}$ & $\mathbf{e l}$ & eu \\
\hline 0.00 & 10.00 & 40.00 & 100.00 & 500.00 & 0.01 & 0.05 & 1.00 & 10.00 & 0.5800 & 0.9800 \\
\hline 0.10 & 11.00 & 39.00 & 110.00 & 480.00 & 0.01 & 0.05 & 1.20 & 9.80 & 0.6310 & 0.9771 \\
\hline 0.20 & 12.00 & 38.00 & 120.00 & 460.00 & 0.01 & 0.05 & 1.40 & 9.60 & 0.6725 & 0.9739 \\
\hline 0.30 & 13.00 & 37.00 & 130.00 & 440.00 & 0.01 & 0.05 & 1.60 & 9.40 & 0.7070 & 0.9704 \\
\hline 0.40 & 14.00 & 36.00 & 140.00 & 420.00 & 0.01 & 0.05 & 1.80 & 9.20 & 0.7363 & 0.9666 \\
\hline 0.50 & 15.00 & 35.00 & 150.00 & 400.00 & 0.01 & 0.05 & 2.00 & 9.00 & 0.7614 & 0.9624 \\
\hline 0.60 & 16.00 & 34.00 & 160.00 & 380.00 & 0.02 & 0.04 & 2.20 & 8.80 & 0.7832 & 0.9578 \\
\hline 0.70 & 17.00 & 33.00 & 170.00 & 360.00 & 0.02 & 0.04 & 2.40 & 8.60 & 0.8024 & 0.9527 \\
\hline 0.80 & 18.00 & 32.00 & 180.00 & 340.00 & 0.02 & 0.04 & 2.60 & 8.40 & 0.8194 & 0.9469 \\
\hline 0.90 & 19.00 & 31.00 & 190.00 & 320.00 & 0.02 & 0.04 & 2.80 & 8.20 & 0.8345 & 0.9405 \\
\hline 1.00 & 20.00 & 30.00 & 200.00 & 300.00 & 0.02 & 0.04 & 3.00 & 8.00 & 0.8480 & 0.9332 \\
\hline
\end{tabular}




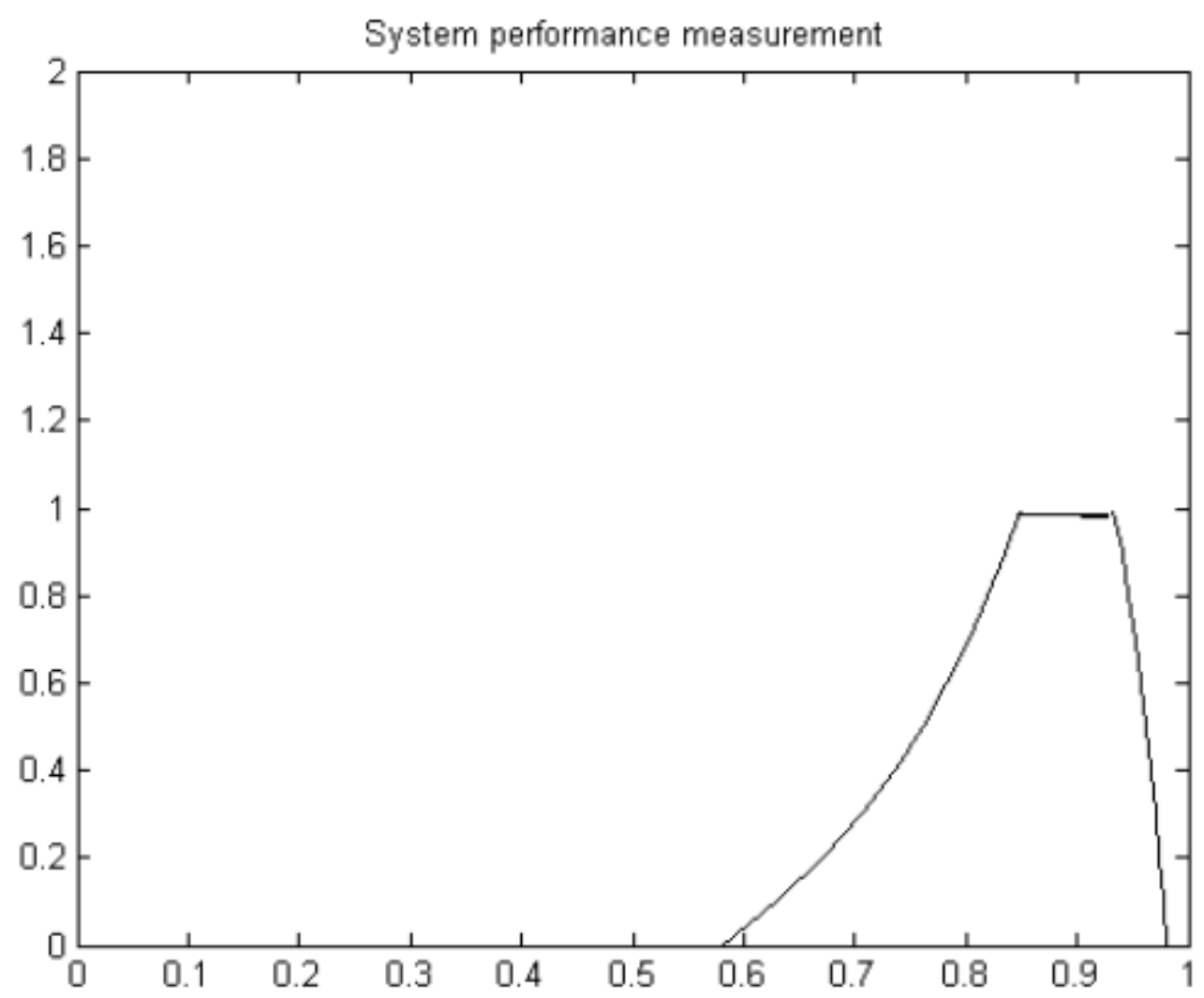

Fig. 2.

\section{Example 3}

If we use Type II FM/FM(FM,FM)/1/ $\infty$ system to model the problem of Example I, the arrival rate, service rate, breakdown rate and repair rate are the same in Example I. The numerical results of optional solutions $\left[\mathrm{F}_{\alpha}^{\mathrm{L}}, \mathrm{F}_{\alpha}^{\mathrm{U}}\right]$ of the membership function of $\tilde{I}$ at different possibilities level $\alpha$ are shown in fig. 3 and table 3.

Table 3

System performance measure of expected idle time

\begin{tabular}{|c|c|c|c|c|c|c|c|c|c|c|}
\hline$\alpha$ & $\mathbf{x} 1$ & $\mathbf{x u}$ & $\mathbf{y l}$ & $\mathbf{y u}$ & $\mathbf{U l}$ & $\mathbf{u u}$ & $\mathbf{v 1}$ & $\mathbf{v u}$ & $\mathbf{e l}$ & $\mathbf{e u}$ \\
\hline 0.00 & 2.00 & 7.00 & 12.00 & 20.00 & 0.05 & 0.50 & 2.00 & 6.00 & 0.2167 & 0.8917 \\
\hline 0.10 & 2.20 & 6.80 & 12.20 & 19.60 & 0.05 & 0.47 & 2.10 & 5.90 & 0.2597 & 0.8785 \\
\hline 0.20 & 2.40 & 6.60 & 12.40 & 19.0 & 0.06 & 0.44 & 2.20 & 5.80 & 0.3011 & 0.8648 \\
\hline 0.30 & 2.60 & 6.40 & 12.60 & 18.80 & 0.07 & 0.41 & 2.30 & 5.70 & 0.408 & 0.8504 \\
\hline 0.40 & 2.80 & 6.20 & 12.80 & 18.40 & 0.07 & 0.38 & 2.40 & 5.60 & 0.3749 & 0.8355 \\
\hline 0.50 & 3.00 & 6.00 & 13.00 & 18.00 & 0.08 & 0.35 & 2.50 & 5.50 & 0.4157 & 0.8199 \\
\hline 0.60 & 3.20 & 5.80 & 13.20 & 17.60 & 0.08 & 0.32 & 2.60 & 5.40 & 0.4510 & 0.8036 \\
\hline 0.70 & 3.40 & 5.60 & 13.40 & 17.20 & 0.09 & 0.29 & 2.70 & 5.30 & 0.4851 & 0.7865 \\
\hline 0.80 & 3.60 & 5.40 & 13.60 & 16.80 & 0.09 & 0.26 & 2.80 & 5.20 & 0.5497 & 0.7500 \\
\hline 1.00 & 4.00 & 5.00 & 14.00 & 16.00 & 0.10 & 0.20 & 3.00 & 5.00 & 0.5804 & 0.7304 \\
\hline
\end{tabular}




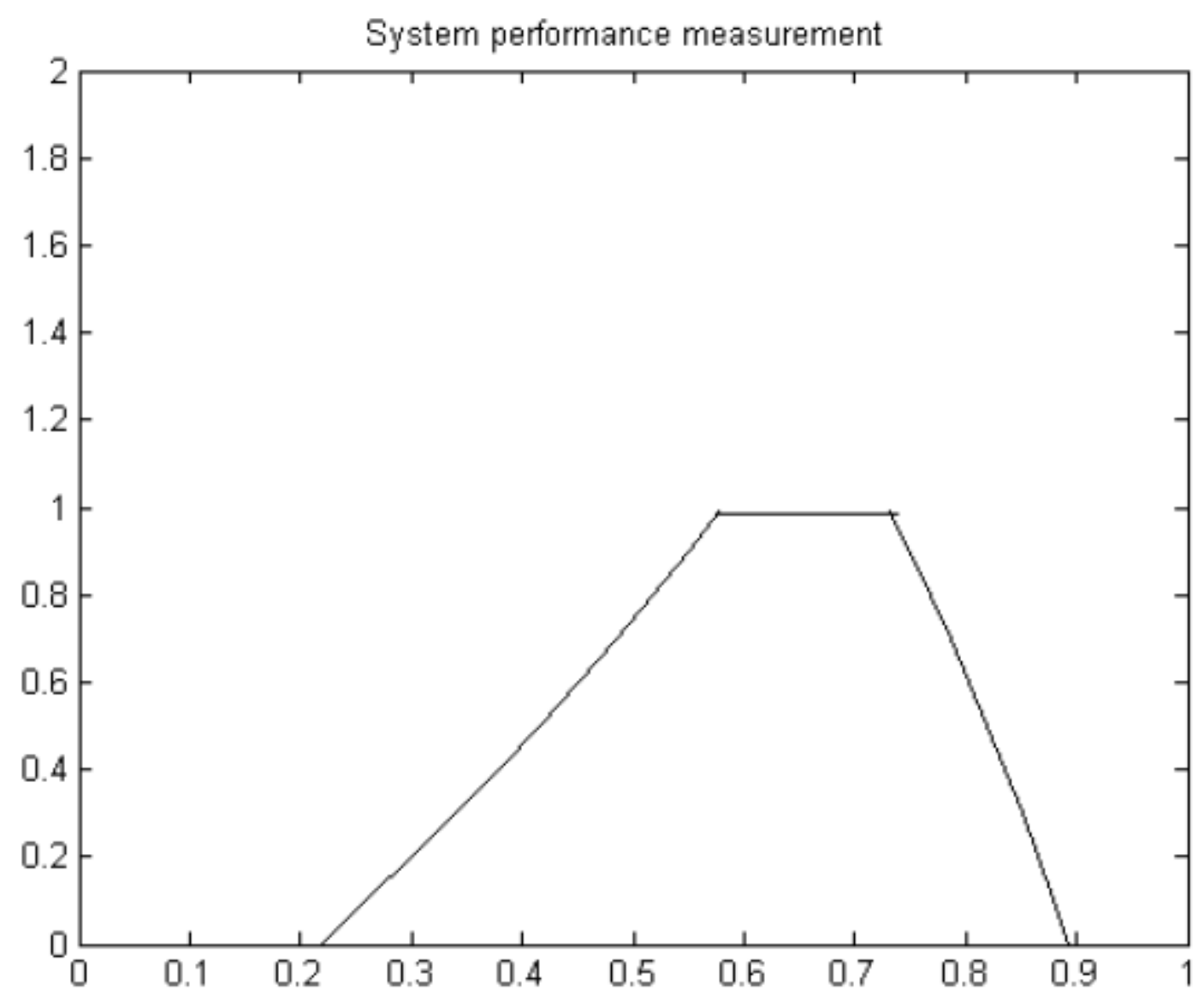

Fig. 3.

By using $\alpha$-cut approach the range of system characteristic at different possibility levels can be obtained. Another point of interest is that there is no direct corresponding behaviour between the membership functions of the system characteristic and the four fuzzy parameters. As seen in Figs. $1-3$, the membership functions of the four fuzzy parameters are trapezoidal fuzzy number, however, the membership functions of the corresponding system characteristic are not trapezoidal.

\section{CONCLUSION}

This paper applied the concept of $\alpha$-cut approach and Zadeh's extension principle to transform a fuzzy queue with an unreliable server into crisp queue with an unreliable server that can be derived by using parametric non-linear programming. Due to complexity of four fuzzy parameters, the closed-form for the corresponding membership function cannot be derived explicitly by taking the inverse of its $\alpha$-cuts at different possibility levels. We therefore presented an efficient algorithm to construct the membership functions of the system chararacteristics. Since the system characteristics are expressed by membership functions rather than crisp values, more information is provided to the management. We successfully investigated the complicated fuzzy queue with an unreliable server and then constructed the membership functions of the system characteristics. These results are significant as well as useful for system designers and practitioners. 


\section{REFERENCES}

[1] J. Bozacatt, J. Shanthikumar, Stochostic models of manufacturing systems, Prentice-Hall, Englewood cliffs, NJ, 1993.

[2] J. Gal, Postoplimal Analysis, Parametric Programming and Related Topics, McGraw-Hill, New York, 1979.

[3] D.P. Gaver, A waiting time with interrupted service, including priorities, Royal statistical society series B 24 (1962) 73-96.

[4] D. Gross, C.M. Harris, Fundamentals of Queuing theory, third ed., John Wiley, New York, 1998.

[5] A. Kaufmann, Introduction to the Theory of Fuzzy subsets, Vol.1, Academic Press, New York, 1075.

[6] W. Li, D. Shi, X. Chao, Reliability analysis of M/G/I queuing systems with server breakdowns and vacations, Journal of Applied probability 34 (1997) 546-555.

[7] H.M. Prade, An outline of fuzzy or possibilistic models for queuing systems, in P.P. wong, S.K. Chang (Eds.), Fuzzy Sets, Plenum Press, New York 1980.

[8] B. Sengupta, A queue with service interruptions in an alternating random environment, operations Research 38 (1990) 308-318.

[9] K.S. Trivedi, probability and Statistics with Reliability, Queuing and Computer Science Applications, John Wiley \& Sons Inc., New York 2002.

[10] K.H. Wang, H.J. Kao, G. Chen, Optimal management of a remarkable and non-reliable server in an infinite and a finite $\mathrm{M} / \mathrm{Hk} / \mathrm{I}$ queuing systems Quality Technology and quantitative Management 1(2) (2004) 325-339.

[11] L.A. Zadeh, Fuzzy sets as a basis for a theory of possibility, fuzzy sets and systems 1 (1978) 328.

[12] H.J. Zimmermann, Fuzzy set Theory and its Applications, 4th ed., Kluor Academic, Bostan, 2001 